\title{
SIMPLE EXPRESSIONS FOR SECOND ORDER DENSITY PERTURBATIONS IN STANDARD COSMOLOGY
}

\author{
Claes Uggla* \\ Department of Physics, \\ University of Karlstad, S-651 88 Karlstad, Sweden \\ JOHN WAINWRIGHT ${ }^{\dagger}$ \\ Department of Applied Mathematics, \\ University of Waterloo, Waterloo, ON, N2L 3G1, Canada
}

\begin{abstract}
In this paper we present four simple expressions for the relativistic first and second order fractional density perturbations for $\Lambda$ CDM cosmologies in different gauges: the Poisson, uniform curvature, total matter and synchronous gauges. A distinctive feature of our approach is the use of a canonical set of quadratic differential expressions involving an arbitrary spatial function, the so-called comoving curvature perturbation, to describe the spatial dependence, which enables us to unify, simplify and extend previous seemingly disparate results. The simple structure of the expressions makes the evolution of the density perturbations completely transparent and clearly displays the effect of the cosmological constant on the dynamics, namely that it stabilizes the perturbations. We expect that the results will be useful in applications, for example, studying the effects of primordial non-Gaussianity on the large scale structure of the universe.
\end{abstract}

PACS numbers: 04.20.-q, 98.80.-k, 98.80.Bp, 98.80.Jk

\section{Introduction}

Increasingly accurate observations of the cosmic microwave background (CMB) and the large scale structure (LSS) of the universe are becoming available and are influencing developments in cosmology. Analyzing these observations necessitates the

*Electronic address: claes.uggla@kau.se

${ }^{\dagger}$ Electronic address: jwainwri@uwaterloo.ca 
theoretical study of possible deviations from linearity, for example, how primordial non-Gaussianity affects the anisotropy of the CMB and the LSS, using nonlinear perturbations of Friedmann-Lemaitre-Robertson-Walker (FLRW) cosmologies (see, e.g [1, 2, 3]). Much of the theoretical work has dealt with nonlinear perturbations of flat FLRW cosmologies with dust (a matter-dominated universe, see e.g [4, 5]) and more recently, also with a cosmological constant ( $\Lambda \mathrm{CDM}$ cosmologies, see e.g [6, 7, 8, 9]). One aspect of the work is to provide an expression for the second order fractional density perturbation ${ }^{(2)} \boldsymbol{\delta}$ that can be used for comparison with observations, and this is the focus of the present paper.

In a previous paper [10] we gave a new expression for ${ }^{(2)} \boldsymbol{\delta}$ in the Poisson gauge, also including the effects of spatial curvature (see equations (33)-(36) in [10]). The evolution of ${ }^{(2)} \boldsymbol{\delta}$ is determined by four functions of time that are integrals involving a Green's function, and these are the main source of the complexity of the expression. Subsequently we realized that if the background spatial curvature is zero (which we will assume in this paper), these functions can be simplified significantly, leading to a physically transparent form of the expression in the Poisson gauge. Then we derived the expressions for ${ }^{(2)} \boldsymbol{\delta}$ in other gauges by using gauge transformation rules. We hope that our final simple expressions will be useful for future work relating LSS observations to theory. The goal of this paper is to give a concise overview of these results in an easily accessible form, leaving the derivations to a separate paper [11], and to use these results to perform an asymptotic analysis. We have made the present paper essentially self-contained, but refer the reader to [11] for full details about the background. Throughout we have made two simplifying restrictions: first that the perturbations at the linear order are purely scalar, and second that the decaying mode is zero.

The outline of the paper is as follows. In the next section we give a unified expression for the first order fractional density perturbation in the four gauges and in section 3 we give the corresponding second order expressions, including the special case when the background cosmology is Einstein-de Sitter. Section 4 contains a discussion of the results, focussing on the dynamics of the perturbations and the effect of the cosmological constant, and the gauge-robustness of the results. A comparison with related work in the literature is also given.

\section{First order perturbations}

The background cosmology is a flat FLRW universe with scale factor $a$ and Hubble scalar $H$, containing dust with background matter density ${ }^{(0)} \rho_{m}$ and a cosmological constant $\Lambda$. The matter-energy content is described by the usual density parameters:

$$
\Omega_{m}=\frac{{ }^{(0)} \rho_{m}}{3 H^{2}}, \quad \Omega_{\Lambda}=\frac{\Lambda}{3 H^{2}},
$$

which satisfy $\Omega_{m}+\Omega_{\Lambda}=11$ We use the dimensionless scale factor $x:=a / a_{0}$, normalized at some reference epoch $t_{0}$, as time variable (when $t_{0}$ is the present time, $x=(1+z)^{-1}$, where $z$ is the redshift). The conservation law shows that $a^{3(0)} \rho_{m}$

\footnotetext{
${ }^{1}$ We use units such that $8 \pi G=1=c$.
} 
is constant, which we write in terms of $\Omega_{m}$ and the dimensionless Hubble scalar $\mathcal{H}:=a H$ as $\mathcal{H}^{2} x \Omega_{m}=m^{2}$. Setting $x=1$ shows that the constant $m$ is given by $m^{2}=\mathcal{H}_{0}^{2} \Omega_{m, 0}$. It follows that

$$
\mathcal{H}^{2}=\mathcal{H}_{0}^{2}\left(\Omega_{\Lambda, 0} x^{2}+\Omega_{m, 0} x^{-1}\right) .
$$

We will use $x, \mathcal{H}, \Omega_{m}$ and the constant $m$ to describe the influence of the background geometry on the perturbations.

We now describe the linear perturbations, subject to the above restrictions. In the Poisson gauge the Bardeen potential is given by:

$$
{ }^{(1)} \Psi_{\mathrm{p}}\left(x, x^{i}\right)=g(x) \zeta\left(x^{i}\right),
$$

where 2

$$
g(x):=\frac{3}{2} m^{2} \frac{\mathcal{H}}{x^{2}} \int_{0}^{x} \frac{d \bar{x}}{\mathcal{H}(\bar{x})^{3}},
$$

and $\zeta\left(x^{i}\right)$ is the linear comoving curvature perturbation, which is exactly conserved for $\Lambda$ CDM cosmologies 3 The fractional density perturbation ${ }^{(1)} \boldsymbol{\delta}_{\bullet}$, where the bullet identifies the gauge, has the following common structure in the Poisson, uniform curvature, total matter and synchronous gauges:

$$
{ }^{(1)} \boldsymbol{\delta} \cdot=A_{\bullet} \zeta+\frac{2}{3} m^{-2} x g \mathbf{D}^{2} \zeta,
$$

where $\mathbf{D}^{2}$ is the spatial Laplacian, defined in eq. (8), while the expressions for $A_{\bullet}$ in the different gauges are as follows:

$$
A_{\mathrm{p}}=-3(1-g), \quad A_{\mathrm{c}}=-3, \quad A_{\mathrm{v}}=A_{\mathrm{s}}=0,
$$

where ${ }_{\mathrm{p}}, \mathrm{c}, \mathrm{v}$ and $\mathrm{s}$ indicate the Poisson, uniform curvature, total matter and synchronous gauges, respectively ((see [11], equation (12)).

\section{Second order perturbations}

We now come to second order perturbations. The second order Bardeen potential in the Poisson gauge is given by

$$
{ }^{(2)} \Psi_{\mathrm{p}}=g\left(\mathcal{B}_{1} \zeta^{2}+\mathcal{B}_{2} \mathcal{D}(\zeta)+\frac{2}{3} m^{-2} x g\left[\mathcal{B}(\mathbf{D} \zeta)^{2}+(1-\mathcal{B}) \mathbf{D}^{2} \mathcal{D}(\zeta)\right]\right),
$$

where $\mathcal{B}_{1}, \mathcal{B}_{2}$ and $\mathcal{B}$ are functions of $x$, and $g$ is the first order perturbation function (4). The spatial dependence is specified by four quadratic differential expressions involving the comoving curvature perturbation $\zeta$, where

$$
(\mathbf{D} \zeta)^{2}:=\gamma^{i j}\left(\mathbf{D}_{i} \zeta\right)\left(\mathbf{D}_{j} \zeta\right), \quad \mathcal{D}(\zeta):=\frac{3}{2} \mathbf{D}^{-4} \mathbf{D}^{i j}\left(\mathbf{D}_{i} \zeta\right)\left(\mathbf{D}_{j} \zeta\right) .
$$

\footnotetext{
${ }^{2}$ See [11], equation (8). The function $g(x)$, up to a constant multiple, is referred to as the growth suppression factor. See for example [8], following equation (11).

${ }^{3}$ See [12, page 15. $\zeta$ is sometimes denoted by $\mathcal{R}$, see for example eq. (7.46) in [13].

${ }^{4}$ See equation (23a) in [11, specialized to the case of a flat background as in section 4.3 in that paper.
} 
Here $\mathbf{D}_{i}$ denotes covariant differentiation with respect to the background spatial metric $\gamma_{i j} 5_{5}$ and $\mathbf{D}^{-2}$ denotes the inverse Laplacian. The Laplacian and the symmetrized trace-free second derivative operator are defined by

$$
\mathbf{D}^{2}:=\gamma^{i j} \mathbf{D}_{i} \mathbf{D}_{j}, \quad \mathbf{D}_{i j}:=\mathbf{D}_{(i} \mathbf{D}_{j)}-\frac{1}{3} \gamma_{i j} \mathbf{D}^{2}
$$

The functions $\mathcal{B}_{1}$ and $\mathcal{B}_{2}$ are algebraic expressions in terms of $g(x)$ :

$$
\begin{aligned}
& \mathcal{B}_{1}(x)=1-2 a_{\mathrm{nl}}-g+\frac{3}{2} \Omega_{m} g^{-1}(1-g)^{2}, \\
& \mathcal{B}_{2}(x)=4(1-g),
\end{aligned}
$$

while $\mathcal{B}$ is an integral involving $g(x)$ and the background variables:

$$
\mathcal{B}(x)=\frac{\mathcal{H}(x)}{2 x^{3} g(x)^{2}} \int_{0}^{x} \frac{\bar{x}^{2} \Omega_{m}}{\mathcal{H}}\left(g^{2}-\frac{3}{2} \Omega_{m}(1-g)^{2}\right) d \bar{x},
$$

where $g, \Omega_{m}$ and $\mathcal{H}$ inside the integral are functions of $\bar{x}$ (see equation (52) in [1]]).

The constant $a_{\mathrm{nl}}$ in (9a) describes the level of primordial non-Gaussianity on super-horizon scales. It enters into the solution of the second order perturbation equations through the values of the metric and matter perturbations at the end of inflation which act as initial conditions by restricting the first and second order conserved quantities ${ }^{7}{ }^{(1)} \zeta_{\mathrm{mw}}:=-{ }^{(1)} \Psi_{\rho}$ and ${ }^{(2)} \zeta_{\mathrm{mw}}:=-{ }^{(2)} \Psi_{\rho}$ according to

$$
{ }^{(2)} \zeta_{\mathrm{mw}}=2 a_{\mathrm{nl}}\left({ }^{(1)} \zeta_{\mathrm{mw}}\right)^{2}
$$

on super-horizon scales. The second order perturbation equations determine ${ }^{(2)} \Psi_{\mathrm{p}}$ up to an arbitrary spatial function $C\left(x^{i}\right)$, which is determined by (11), thereby introducing $a_{\mathrm{nl}}$ into the solution. The absence of primordial non-Gaussianity corresponds to $a_{\mathrm{nl}}=1$. We refer to section 3.5 in [11] and other references given there for details.

The functions $g(x)$ and $\mathcal{B}(x)$, which determine the time dependence of ${ }^{(2)} \Psi_{\mathrm{p}}$, also determine the time dependence of the second order fractional density perturbation ${ }^{(2)} \boldsymbol{\delta}$. In order to give a unified description of the spatial dependence of ${ }^{(2)} \boldsymbol{\delta}$ • we introduce the following eight 'canonical' quadratic differential expressions:

$$
\zeta^{2}, \quad \mathcal{D}(\zeta), \quad(\mathbf{D} \zeta)^{2}, \quad \mathbf{D}^{2} \mathcal{D}(\zeta), \quad \mathbf{D}^{2} \zeta^{2}, \quad \mathbf{D}^{2}(\mathbf{D} \zeta)^{2}, \quad \mathbf{D}^{4} \mathcal{D}(\zeta), \quad\left(\mathbf{D}^{2} \zeta\right)^{2},
$$

the first four of which appear in ${ }^{(2)} \Psi_{\mathrm{p}}$. The first seven of these determine the spatial

\footnotetext{
${ }^{5}$ In the present case $\gamma_{i j}$ is the Euclidian metric, which in Cartesian coordinates yields $\mathbf{D}_{i}=\partial_{i}$. However, since [10] and [11] also deal with non-zero background spatial curvature, we keep the notation of those papers.

${ }^{6}$ The expression (6) for ${ }^{(2)} \Psi_{\mathrm{p}}$ includes the initial conditions and is given by equations (23a) and (38) in [11, with $C_{\mathrm{nl}}$ given by (42). Thus the functions (9) are obtained from $\mathcal{B}_{1}(x)$ and $\mathcal{B}_{1}(x)$ in [11] when $K=0$, as given by equations $(45 \mathrm{c}, \mathrm{d})$, by adding $\frac{4}{5}-2 a_{\mathrm{nl}}$ and $\frac{8}{5}$, respectively.

${ }^{7}$ See 13 , equations (7.61) and (7.71). The subscript $\rho$ stands for the uniform density gauge, and the subscript mw stands for Malik-Wands.
} 
dependence of ${ }^{(2)} \boldsymbol{\delta}$. in the Poisson, uniform curvature and total matter gauges:

$$
\begin{aligned}
{ }^{(2)} \boldsymbol{\delta} \bullet & \underbrace{A_{1}, \zeta^{2}+A_{2, \boldsymbol{\bullet}} \mathcal{D}(\zeta)}_{\text {the super-horizon part }} \\
& +\underbrace{\frac{2}{3} m^{-2} x g\left[A_{3, \bullet}(\mathbf{D} \zeta)^{2}+A_{4, \boldsymbol{\bullet}} \mathbf{D}^{2} \mathcal{D}(\zeta)+A_{5, \boldsymbol{\bullet}} \mathbf{D}^{2} \zeta^{2}\right]}_{\text {the post-Newtonian part }} \\
& +\underbrace{\frac{4}{9} m^{-4} x^{2} g^{2}\left[\mathcal{B} \mathbf{D}^{2}(\mathbf{D} \zeta)^{2}+(1-\mathcal{B}) \mathbf{D}^{4} \mathcal{D}(\zeta)\right]}_{\text {the Newtonian part }},
\end{aligned}
$$

where the bullet indicates the gauge. The time dependence is contained in the coefficients $\mathcal{B}$ and $A_{i, \bullet}, i=1, \ldots, 5$, which are functions of the scale factor $x$. The three labeled parts in (13) are identified by the weight of the operator $\mathbf{D}_{i}$ acting on $\zeta\left(x^{i}\right)$. For the super-horizon part, $\mathbf{D}_{i}$ has weight zero, for the post-Newtonian part it has weight 2 , and for the Newtonian part it has weight 4.

We obtain the coefficients $A_{i, \bullet}$ in (13) by setting the background spatial curvature to zero $(K=0)$ in the general expressions in [11] (see section 4.3 in that paper for details). In the Poisson gauge we have

$$
\begin{aligned}
& A_{1, \mathrm{p}}=3(1-g)\left(1+2 a_{\mathrm{nl}}-4 g+\frac{3}{2} \Omega_{m}(1-g)\right), \\
& A_{2, \mathrm{p}}=12 g(1-g), \\
& A_{3, \mathrm{p}}=3 g(\mathcal{B}-2)-\frac{3}{2} \Omega_{m} g^{-1}(1-g)^{2}, \\
& A_{4, \mathrm{p}}=-2-3 g(\mathcal{B}-1)-\frac{3}{2} \Omega_{m} g^{-1}(1-g)^{2}, \\
& A_{5, \mathrm{p}}=1-2 a_{\mathrm{nl}}+3 g+\frac{3}{2} \Omega_{m} g^{-1}(1-g)^{2},
\end{aligned}
$$

in the uniform curvature gauge,

$$
\begin{aligned}
& A_{1, \mathrm{c}}=3\left(1+2 a_{\mathrm{nl}}\right), \\
& A_{2, \mathrm{c}}=0, \\
& A_{3, \mathrm{c}}=-\frac{3}{2} \Omega_{m} g^{-1}, \\
& A_{4, \mathrm{c}}=-2-\frac{3}{2} \Omega_{m} g^{-1}(1-2 g), \\
& A_{5, \mathrm{c}}=1-2 a_{\mathrm{nl}}+\frac{3}{2} \Omega_{m} g^{-1}(1-g),
\end{aligned}
$$

and in the total matter gauge,

$$
\left(A_{1}, A_{2}, A_{3}, A_{4}, A_{5}\right)_{\mathrm{v}}=\left(0,0,-5,0,2\left(2-a_{\mathrm{nl}}\right)\right) .
$$

For the synchronous gauge we have (equation (55) in [11])

$$
\begin{aligned}
{ }^{(2)} \boldsymbol{\delta}_{\mathrm{s}}= & \frac{2}{3} m^{-2} x g\left[-5(\mathbf{D} \zeta)^{2}+2\left(2-a_{\mathrm{nl}}\right) \mathbf{D}^{2} \zeta^{2}\right] \\
& +\frac{4}{9} m^{-4} x^{2} g^{2}\left[\left(\mathcal{B}+\frac{1}{3}\right)\left(\mathbf{D}^{2}(\mathbf{D} \zeta)^{2}-\mathbf{D}^{4} \mathcal{D}(\zeta)\right)+2\left(\mathbf{D}^{2} \zeta\right)^{2}\right]
\end{aligned}
$$

Note the additional term $\left(\mathbf{D}^{2} \zeta\right)^{2}$ in the Newtonian part, which modifies the form (13) . 


\subsection{Einstein-de Sitter as background}

In the limiting case $\Lambda=0$ the background is the Einstein-de Sitter universe, which satisfies $\Omega_{m}=1, \Omega_{\Lambda}=0$ and $\mathcal{H}^{2} x=m^{2}$. It follows from (4) and (10) that the key functions $g(x)$ and $\mathcal{B}(x)$ simplify to

$$
g(x)=\frac{3}{5}, \quad \mathcal{B}(x)=\frac{1}{21} .
$$

Substituting (18) into (14), (15) and (16) shows that ${ }^{(2)} \boldsymbol{\delta}$ • has the following form for the Poisson, uniform curvature and total matter gauges:

$$
\begin{aligned}
{ }^{(2)} \boldsymbol{\delta}_{\bullet}= & A_{1, \bullet} \zeta^{2}+A_{2, \bullet} \mathcal{D}(\zeta) \\
& +\frac{2}{5} m^{-2} x\left[A_{3, \bullet}(\mathbf{D} \zeta)^{2}+A_{4, \bullet} \mathbf{D}^{2} \mathcal{D}(\zeta)+A_{5, \bullet} \mathbf{D}^{2} \zeta^{2}\right] \\
& +\frac{4}{25} m^{-4} x^{2}\left[\frac{1}{21} \mathbf{D}^{2}(\mathbf{D} \zeta)^{2}+\frac{20}{21} \mathbf{D}^{4} \mathcal{D}(\zeta)\right]
\end{aligned}
$$

where the coefficients $A_{i, \bullet}$ specialize to

$$
\begin{aligned}
& \left(A_{1}, A_{2}, A_{3}, A_{4}, A_{5}\right)_{\mathrm{p}}=\left(-\frac{12}{5}\left(\frac{2}{5}-a_{\mathrm{nl}}\right), \frac{72}{25},-\frac{137}{35},-\frac{24}{35}, 2\left(\frac{8}{5}-a_{\mathrm{nl}}\right)\right), \\
& \left(A_{1}, A_{2}, A_{3}, A_{4}, A_{5}\right)_{\mathrm{c}}=\left(3\left(1+2 a_{\mathrm{nl}}\right), 0,-\frac{5}{2},-\frac{3}{2}, 2\left(1-a_{\mathrm{nl}}\right)\right), \\
& \left(A_{1}, A_{2}, A_{3}, A_{4}, A_{5}\right)_{\mathrm{v}}=\left(0,0,-5,0,2\left(2-a_{\mathrm{nl}}\right)\right) .
\end{aligned}
$$

For the synchronous gauge, using (17) and (18), we obtain

$$
\begin{aligned}
{ }^{(2)} \boldsymbol{\delta}_{\mathrm{s}}= & \frac{2}{5} m^{-2} x\left[-5(\mathbf{D} \zeta)^{2}+2\left(2-a_{\mathrm{nl}}\right) \mathbf{D}^{2} \zeta^{2}\right] \\
& +\frac{4}{25} m^{-4} x^{2}\left[\frac{8}{21}\left(\mathbf{D}^{2}(\mathbf{D} \zeta)^{2}-\mathbf{D}^{4} \mathcal{D}(\zeta)\right)+2\left(\mathbf{D}^{2} \zeta\right)^{2}\right]
\end{aligned}
$$

This case has a lengthy history, starting with Tomita [14]. Expressions for ${ }^{(2)} \boldsymbol{\delta}$ • in the synchronous gauge have been given in [14] (eq. (5.1)), 4] (eq. (4.33)) and [5] (eq. (7)), and in the Poisson gauge in [4] (eq. (6.10)), 5] (eq. (8)) and [15] (eq. (43)). The different expressions are not related in an obvious way. However, after introducing our canonical quadratic differential quantities (12), we find agreement with our expression for ${ }^{(2)} \boldsymbol{\delta}_{\mathrm{s}}$, except that [4] appears to differ by an overall factor of $\frac{1}{2}$. Further, as regards the Poisson gauge we find complete agreement with [5] and [15], but encounter discrepancies with numerical coefficients in [4].

\section{Discussion}

In this paper we have given simple expressions for the second order fractional density perturbation ${ }^{(2)} \boldsymbol{\delta}$. for flat FLRW cosmologies containing dust and a cosmological constant in four gauges: Poisson, uniform curvature, total matter and synchronous. The unified form of our expressions is due to our novel way of representing the temporal and spatial dependence. First, as regards temporal dependence the expressions depend algebraically on the function $g(x)$ that determines the first order perturbations and on one other function $\mathcal{B}(x)$ that is defined as an integral involving $g(x)$ and the background variables (see eq. (10) ). Second, we have introduced a new way of representing the spatial dependence of the perturbations using the set of quadratic differential expressions given in (12). 
Our representation shows that ${ }^{(2)} \boldsymbol{\delta}$ • assumes its simplest form in the total matter and synchronous gauges, with the super-horizon part being zero and the terms in the post-Newtonian part having a common time dependence of $x g(x)$. On the other hand, the form in the Poisson gauge is the most complicated. For example, the function $\mathcal{B}(x)$ appears in the post-Newtonian part as well as in the Newtonian part, unlike in the other gauges.

The form of our expressions for ${ }^{(2)} \boldsymbol{\delta}$. makes it easy to understand their overall dynamics and to read off their asymptotic behaviour near the initial singularity $(x \rightarrow 0)$ and at late times $(x \rightarrow \infty)$. It follows from (4) and (10) that

$$
\lim _{x \rightarrow 0} g(x)=\frac{3}{5}, \quad \lim _{x \rightarrow 0} \mathcal{B}(x)=\frac{1}{21} .
$$

In addition if $\Lambda>0$ then (2) yields $\mathcal{H}=O(x)$ and $\Omega_{m}=O\left(x^{-3}\right)$ as $x \rightarrow \infty$, which implies that

$$
\lim _{x \rightarrow \infty} x g(x) \quad \text { and } \quad \lim _{x \rightarrow \infty} \mathcal{B}(x) \quad \text { are finite and non-zero. }
$$

Using these results, and the definitions (44) and (10), we conclude that $g(x), \mathcal{B}(x)$ and $g^{-1} \Omega_{m}$ are positive and bounded for $0<x<\infty$, and hence that the coefficients $A_{i, \bullet}$, as given by (14)-(16), are bounded. It follows from (13) and (17) that the overall evolution of ${ }^{(2)} \boldsymbol{\delta}_{\bullet}$ is governed by the factors $x g$ and $x^{2} g^{2}$ in the post-Newtonian and Newtonian terms.

As regards asymptotics, equations (18) and (22) imply that near the initial singularity ${ }^{(2)} \boldsymbol{\delta}$ • is approximated by the Einstein-de Sitter specialization (19)-(21). It

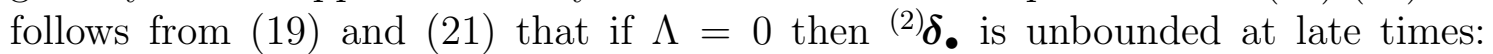
${ }^{(2)} \boldsymbol{\delta}_{\bullet}=O\left(x^{2}\right)$ as $x \rightarrow \infty$, with the Newtonian part being dominant. In conjunction with the linear behaviour, ${ }^{(1)} \boldsymbol{\delta}_{\bullet}=O(x)$ as $x \rightarrow \infty$, this suggests that the Einsteinde Sitter universe is unstable to scalar perturbations. On the other hand, if $\Lambda>0$ eq. (23), in conjunction with (13)-(17), implies that both ${ }^{(1)} \boldsymbol{\delta}$ • and ${ }^{(2)} \boldsymbol{\delta}$ • are finite for all four gauges when $x \rightarrow \infty$. This suggests that the presence of a positive cosmological constant stabilizes the perturbations, and that this is the case for any of the four gauges under consideration. We can thus say that the conclusion reached in [10] (see equations (2) and (3)) concerning stabilization is gauge-robust. However, the choice of gauge does affect the limit of ${ }^{(2)} \boldsymbol{\delta}$. as $x \rightarrow \infty$. Specifically it follows from (14)-(17) and the above-mentioned asymptotic properties of $g$ and $\Omega_{m}$ that

$$
\lim _{x \rightarrow \infty}\left(A_{1}, A_{2}, A_{3}, A_{4}, A_{5}\right) \bullet=\left(3\left(1+2 a_{\mathrm{nl}}\right), 0,0,-2,1-2 a_{\mathrm{nl}}\right),
$$

for the Poisson and uniform curvature gauges and

$$
\lim _{x \rightarrow \infty}\left(A_{1}, A_{2}, A_{3}, A_{4}, A_{5}\right) \bullet=\left(0,0,-5,0,2\left(2-a_{\mathrm{nl}}\right)\right),
$$

for the total matter and synchronous gauges. Observe that the effect of the nonGaussianity, as represented by $a_{\mathrm{nl}}$, persists indefinitely into the future through the post-Newtonian term and in the first case also through the super-horizon term.

In the course of doing the research reported in this paper we made a detailed comparison of our results with the known expressions for ${ }^{(2)} \boldsymbol{\delta} \bullet$ in the synchronous and 
Poisson gauges when the background spatial curvature is zero. Our new canonical representation of the spatial dependence has enabled us to unify, simplify and extend seemingly disparate results, while at the same time revealing a number of errors in the literature. One example concerns the Poisson gauge. Two expressions for ${ }^{(2)} \boldsymbol{\delta}_{\mathrm{p}}$ with $\Lambda>0$ have been given, which appear to be completely different. The first, by Tomita [6], was derived by solving the perturbation equations in the synchronous gauge and then transforming to the Poisson gauge. The second, by Bartolo et al [8], was derived by solving the perturbation equations directly in the Poisson gauge. However, by simplifying the $B$-functions of Bartolo and introducing our canonical representation of the spatial dependence we have been able to show, after correcting some typos, that both of these expressions can be written in our form for ${ }^{(2)} \boldsymbol{\delta}_{\mathrm{p}}$, which is given by (13) and (14).

Another example concerns the synchronous gauge. Three expressions for ${ }^{(2)} \boldsymbol{\delta}_{\mathrm{s}}$ with $\Lambda>0$ have been given [6, 8, 9], which are not related in an obvious way. We have shown that the expression given by Tomita [6] can be written in our form, given by (17). As regards [8] and [9] the post-Newtonian part can be written in our form, but in [8] the Newtonian term is an approximation, while in [9] the temporal and spatial dependence is not given in fully explicit form.

To the best of our knowledge an expression for ${ }^{(2)} \boldsymbol{\delta}$ • with $\Lambda>0$ has not been given previously for gauges other than the Poisson and synchronous gauges. However, Hwang et al [15] have given an expression for ${ }^{(2)} \boldsymbol{\delta}$ • with $\Lambda=0$ using the total matter and uniform curvature gauges (see equations (43) and (53)). After converting the spatial dependence to our canonical form we obtain agreement with our expressions. Details about all the above comparisons are given in [11].

\section{Acknowledgements}

We thank Marco Bruni and David Wands for helpful correspondence concerning their recent paper [9]. CU also thanks the Department of Applied Mathematics at the University of Waterloo for kind hospitality. JW acknowledges financial support from the University of Waterloo.

\section{References}

[1] N. Bartolo, E. Komatsu, S. Matarrese and A. Riotto. Non-Gaussianity from inflation: theory and observations, Physics Reports 402, 103-266 (2004).

[2] N. Bartolo, S. Matarrese and A. Riotto. Non-Gaussianity and the Cosmic Microwave Background Anisotropies. Advances in Astronomy, 2010, 157079, (2010), arXiv:1001.3957 [astro-ph.CO].

[3] C. Pitrou, J-P. Uzan, and F. Bernardeau. The cosmic microwave background bispectrum from the non-linear evolution of the cosmological perturbations. JCAP 1007, 003 (2010).

[4] S. Matarrese, S. Mollerach and M. Bruni. Relativistic second-order perturbations of the Einstein-de Sitter universe. Phys. Rev. D 58, 043504 (1998). 
[5] N. Bartolo, S. Matarrese and A. Riotto. Signatures of Primordial NonGaussianity in the Large-Scale Structure of the Universe. JCAP 0510, 010 (2005).

[6] K. Tomita. Relativistic second-order perturbations of nonzero- $\Lambda$ flat cosmological models and CMB anisotropies. Phys. Rev. D 71, 083504 (2005).

[7] N. Bartolo, S. Matarrese and A. Riotto. The full second-order radiation transfer function for large-scale CMB anisotropies. JCAP 0605, 010 (2006).

[8] N. Bartolo, S. Matarrese, O. Pantano and A. Riotto. Second-order matter perturbations in a $\Lambda \mathrm{CDM}$ cosmology and non-Gaussianity. Class. Quantum Grav. 27, 124009 (2010).

[9] M. Bruni, J. C. Hidalgo, N. Meures and D. Wands. Non-Gaussian initial conditions in $\Lambda \mathrm{CDM}$ : Newtonian, relativistic and primoridial contributions. arXiv:1307.1478v1.

[10] C. Uggla and J. Wainwright. Asymptotic analysis of perturbed dust cosmologies to second order. Gen. Rel. Grav. 451467 (2013) doi:10.1007/s10714-013-1559-0.

[11] C. Uggla and J. Wainwright. Second order density perturbations for dust cosmologies. arXiv:0910136

[12] C. Uggla and J. Wainwright. Scalar Cosmological Perturbations. Class. Quantum Grav. 29105002 (2012) doi:10.1088/0264-9381/29/10/105002.

[13] K. A. Malik and D. Wands. Cosmological perturbations. Physics Reports 475, $1-51$ (2009).

[14] K. Tomita. Non-linear theory of gravitational instability in an expanding universe. Prog. Theor. Phys. 37, 831 (1967).

[15] J-C. Hwang, H. Noh, and J-O. Gong. Second order solutions of cosmological perturbation in the matter dominated era. Astrophys. J. 752, 50 (2012) doi:10.1088/0004-637X/752/1/50. 